\begin{tabular}{|c|l|}
\hline Title & $\begin{array}{l}\text { Nonresonant femtosecond second hy perpolarizabilities of intramolecular charge transfer molecules with great excited- } \\
\text { and ground-state dipole moment differences }\end{array}$ \\
\hline Author(s) & Y amashita, Mikio; Kikuma, Shinji; Y amaoka, Y oshihisa; Murakami, Hidetoshi; Morita, Ry uji; Shigekawa, Hidemi \\
\hline Citation & A pplied Physics Letters, 75(1), 28-30 \\
\hline Issue Date & 1999-07-05 \\
\hline Doc URL & http://hdl.handle.net/2115/6078 \\
\hline Rights & Copyright $\odot 1999$ A merican Institute of Physics \\
\hline Type & article \\
\hline File Information & APL 75-1.pdf \\
\hline
\end{tabular}

Instructions for use 


\title{
Nonresonant femtosecond second hyperpolarizabilities of intramolecular charge-transfer molecules with great excited- and ground-state dipole-moment differences
}

\author{
Mikio Yamashita, ${ }^{\text {a) }}$ Shinji Kikuma, Yoshihisa Yamaoka, \\ Hidetoshi Murakami, and Ryuji Morita \\ Department of Applied Physics, Hokkaido University, Kita-13, Nishi-8, Kita-Ku, Sapporo 060-8628, Japan \\ and CREST, Japan Science and Technology Corporation (JST) \\ Hidemi Shigekawa \\ Institute of Materials Science, University of Tsukuba and Center for Tsukuba Advanced Research Alliance \\ (TARA), Tsukuba 305.8573, Japan \\ and CREST, Japan Science and Technology Corporation (JST)
}

(Received 3 December 1998; accepted for publication 23 April 1999)

\begin{abstract}
The femtosecond second hyperpolarizability due to the nonresonant electronic polarization for two intramolecular charge-transfer molecules, 6-propionyl-2-dimethylamino naphthalene and 2 -anilinonaphthalene-6-sulfonic acid which possess the great permanent dipole-moment difference between the excited state and the ground state, is obtained from the measurement of the ultrafast change in refractive index using a time-resolved interferometer. It is found that the second hyperpolarizability increases parabolically from $5 \times 10^{-34}$ to $4.3 \times 10^{-32}$ esu with increasing the dipole-moment difference from 7.7 to $40 \mathrm{D}$, and this quantitative relationship is well explained by a quantum-mechanical equation describing the electronic second hyperpolarizability of one molecule with the permanent dipole-moment difference. (c) 1999 American Institute of Physics.
\end{abstract} [S0003-6951(99)02025-2]

Recently, highly third-order nonlinear optical materials with a femtosecond response and no absorption have come to be desired for the development of the ultrafast opticalcontrol devices. ${ }^{1}$ One of the materials satisfying these optical properties is anticipated to be the organic compound having the nonlinearity caused by intramolecular charge transfer (ICT) in the nonresonant region. ${ }^{2}$ However, the investigation of the femtosecond nonresonant dynamics of the third-order optical nonlinearity for molecules possessing the large ICT hardly has been carried out so far.

In this letter, we report that ICT molecules, with the great difference $|\Delta \mu|=\left|\mu_{e e}-\mu_{g g}\right|(\geqslant 20 D)$ between the permanent electric dipole moment of the excited state $\mu_{e e}$ and that of the ground state $\mu_{g g}$, have the highly nonlinear electronic polarization even in the nonresonant region, and the third-order nonlinearity responds instantaneously within the duration of the femtosecond pump pulse. Furthermore, it is found experimentally that the angular-averaged second hyperpolarizability $\langle\gamma\rangle$ of the molecule indicates a parabolic dependence as an increasing function of the dipole-moment difference $|\Delta \mu|$ over the wide range from $|\Delta \mu| \cong 5$ to $40 \mathrm{D}$.

The large ICT compounds studied in this investigation are 6-propionyl-2-dimethylamino naphthalene (PRODAN) and 2-anilinonaphthalene-6-sulfonic acid (2,6-ANS). They were obtained from Molecular Probes, Inc. Their ultrafast third-order nonlinear optical responses were examined for the sample solutions dissolved in ethanol (EtOH) at the dilute concentration. The absorption peak of the PRODAN solution is at $360 \mathrm{~nm}$ with the long-wavelength absorption edge

\footnotetext{
${ }^{4)}$ Electronic mail: mikio@eng.hokudai.ac.jp
}

around $430 \mathrm{~nm}$, where the molar absorption coefficient is $1.6 \%$ of the maximum one at $360 \mathrm{~nm}$ (the maximum molar absorption coefficient of $18400 \mathrm{~cm}^{2} / \mathrm{mM}$, the half width of $2525 \mathrm{~cm}^{-1}$ and the electronic transition dipole moment of 4.5 D). ${ }^{3}$ The absorption peak of the 2,6 -ANS solution is at $330 \mathrm{~nm}$ with the long-wavelength absorption edge around $397 \mathrm{~nm}$ where the molar absorption coefficient is $1.6 \%$ of the maximum one (the maximum molar absorption coefficient of $\left.50880 \mathrm{~cm}^{2} / \mathrm{mM}\right)$.

Since PRODAN has a good electron donor of the dimethyl amino group and a good electron acceptor of the pro pionyl group, attached to the 2 and 6 positions of the large naphthalene ring, it shows a great charge-transfer character. The dipole-moment difference $|\Delta \mu|$ between the first excited electronic state and the ground state is known to be $20 \mathrm{D}$ on the basis of the established measurement of the Stokes shift of the absorption and emission spectra. ${ }^{3}$ Similarly, 2,6-ANS has a good electron donor of the $N$-phenyl amino group and a good electron acceptor of the sulfonate group attached to the 2 and 6 positions of the naphthalene ring, and hence indicates a large $|\Delta \mu|=40 \mathrm{D} .^{4}$

The apparatus for the measurement of the femtosecond time-dependent third-order nonlinearity using a timeresolved interferometer with a heterodyne pump and probe technique is the same as the previously employed one, except for some points. ${ }^{5,6} 100 \mathrm{fs}$ pulses at $780 \mathrm{~nm}$ with the average power of $850 \mathrm{~mW}$ at a $100 \mathrm{MHz}$ repetition rate were generated from a Kerr-lens mode-locked Tissapphire laser: The linearly polarized laser output beam was split in a strong pump-pulse beam and a weak pulse beam $(9: 1)$. The latter beam, furthermore, was split in a probe pulse beam and a reference pulse beam (6:4) to compose of the time-division 


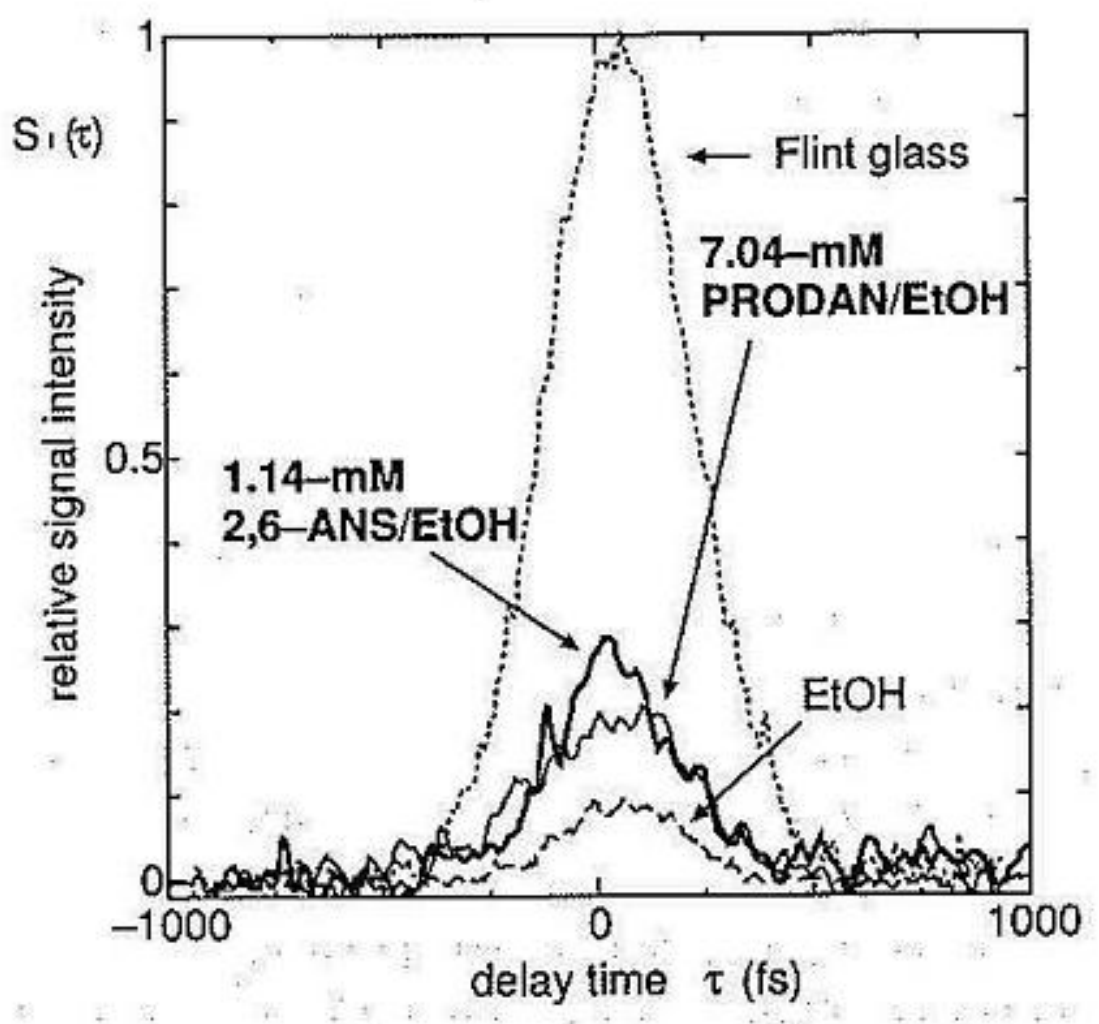

FIG. 1. Phase-change signals from $7.04 \mathrm{mM}$ PRODAN solution (solid line), $1.14 \mathrm{mM}$ 2,6-ANS solution (thick solid line), flint glass as a reference (dotted line) and ethanolic solvent (dashed line).

interferometer. The carrier frequency of the probe pulse was up-shifted by $39.0 \mathrm{MHz}$ using an acousto-optic modulator, and that of the reference pulse was also up-shifted by 40.0 $\mathrm{MHz}$ using another modulator. Their diffraction efficiencies were $60 \%-65 \%$, and their linear polarizations were parallel to each other. Both the pulse durations were broadened to 385 fs due to group-velocity dispersion of the materials of acousto-optic modulators. The probe pulse was time delayed ( $\tau$ ) with respect to the $1.14 \mathrm{kHz}$ chopped pump pulse by a displacementmeter-attached stepping motor. After the advanced reference pulse ( $330 \mathrm{ps}$ ahead of the probe pulse) and the probe pulse were copropagated, these pulses and the pump pulse were noncollinearly focussed on the sample solution in a 1-mm-thick quartz cell (or a reference sample of a high-refractive-index flint glass with a $1.2 \mathrm{~mm}$ thickness) by a $10 \mathrm{~cm}$ focusing lens. Passing through the sample, the pump-pulse-induced phase-change probe pulse and the reference pulse were collimated by another $10 \mathrm{~cm}$ focusing lens, and overlapped in space and time at a fast response photodiode. The $1 \mathrm{MHz}$ beat signal of the probe-reference pulses was detected by an FM ham-radio receiver. The output yields an electric signal proportional to the derivative of the probe-pulse phase change with respect to the time. Since the pump pulse was chopped at $1.14 \mathrm{kHz}$, the receiver output was modulated at the same frequency. By integrating the modulated output through a lock-in amplifier, the probepulse phase change was measured at each delay time $\tau$.

Figure 1 shows the typical signal $S_{I I E}(\tau)$ of the femtosecond time-dependent phase change (solid line) in the nonresonant region for the $7.04 \mathrm{mM}$ dilute solution of PRODAN and the signal $S_{I I E}(\tau)$ (thick solid line) for the $1.14 \mathrm{mM}$ dilute solution of 2,6 -ANS. In addition, the signal $S_{R}(\tau)$ (dotted line) for the reference flint glass and the signal $S_{E}(\tau)$ (dashed line) for the ethanolic solvent are shown. The reference signal was measured two times before and after the measurements of the sample signal and the solvent signal, and its averaged result is shown in Fig. 1. Both the signals of the two ICT-molecule solutions respond instantaneously within $385 \mathrm{fs}$ durations of the probe and reference pulses, as well as the case of the reference glass which is already
TABLE I. Third-order nonlinear optical parameters and permanent electric dipole-moment differences between excited and ground states for different ICT molecules.

\begin{tabular}{|c|c|c|c|}
\hline & $\left|x i^{(3)}\right|($ esu) & $\left\langle\gamma_{1}\right\rangle$ (esu) & $1 \Delta \mu \mid(0)$ \\
\hline$\underset{\substack{\text { PRODAN } \\
(7.04 \mathrm{mM})_{\mathrm{CH}} \mathrm{CH}_{2} \mathrm{C}}}{\mathrm{O}} \underset{\mathrm{O}}{\mathrm{N}(\mathrm{CHs})_{2}}$ & $5.7 \times 10^{34}$ & $4.9 \times 10^{-33}$ & 20 \\
\hline${ }_{(1.14 \mathrm{mM})}^{2,6 \text {-ANS }}{ }_{\mathrm{HO}_{3} \mathrm{~S}} \widehat{\mathrm{O}} \widehat{\mathrm{O}}^{\mathrm{H}}-\mathrm{O}$ & $8.1 \times 10^{-14}$ & $4.3 \times 10^{32}$ & 40 \\
\hline $\begin{array}{l}\text { DEANST } \\
\quad\left(\mathrm{CH}_{3} \mathrm{CH} 2\right)_{2} \mathrm{~N}-(\mathrm{O})-\mathrm{CH}=\mathrm{CH}-\mathrm{NO} 2\end{array}$ & & $5 \times 10^{-3}$ & 7.7 \\
\hline
\end{tabular}

known to indicate the instantaneous response. ${ }^{5,7,8}$ This result of the instantaneous change in refractive index in the femtosecond time region suggests that the nonlinear response measured for the two ICT-molecule solutions originates from only the nonlinear electronic polarization in the off-resonant region (not from the polarization due to the molecular motion and/or the intermolecular interaction such as the thiophene solution). ${ }^{7,9,10}$ The third-order nonlinear electronic susceptibility $|\chi\rangle^{(3)} \mid$ of only ICT molecules at the measured concentration is evaluated from the following equation for the nonlinear response faster than the pulse employed for the measurement: ${ }^{5,7,11,12}$

$$
\left|\chi_{I}^{(3)}\right|=\left\{n_{0 / I E}^{2} L_{R}\left|\chi_{R}^{(3)}\right| /\left(n_{0 R}^{2} L_{I / E}\right)\right\} \times\left(S_{I}^{T} / S_{R}^{T}\right),
$$

where the integral signals are $S_{i}^{T}=\int_{-\infty}^{\infty} S_{i}(\tau) d \tau$ for the sample solutions $\left[i=I / E\right.$ for $\left.S_{I / E}(\tau)\right]$, the ethanolic solvent $\left[i=E\right.$ for $\left.S_{E}(\tau)\right]$ and the reference glass $\left[i=R\right.$ for $\left.S_{R}(\tau)\right]$, $S_{I}^{T}=S_{l / E}^{T}-S_{E}^{T}$, the linear refractive indices are $n_{0 / / E}=1.362$ for the sample solutions and $n_{0, R}=2.148$ for the reference glass, and the sample and reference lengths are $L_{I I E}$ $=1.0 \mathrm{~mm}$ and $L_{R}=1.2 \mathrm{~mm}$. The optical density (OD) of the $1 \mathrm{~mm}$ sample solution at the measured wavelength of 780 $\mathrm{nm}$ is unmeasurably small (OD $<0.01)$. The subscripts $I / E$, $E$ and $R$ refer to the sample solution, the ethanolic solvent and the reference glass, respectively. The third-order nonlinear susceptibility $\left|\chi_{R}^{(3)}\right|$ of the reference flint glass with isotropic structure is $10.3 \times 10^{-13} \mathrm{esu}$ in the nonresonant region. $5,7,8$

The use of the equation relating the $\left|\chi_{I}^{(3)}\right|$ to the molecular second hyperpolarizability, $\left|\chi_{I}^{(3)}\right|=N\left\{\left(n_{0 H / E}^{2}\right.\right.$ $+2) / 3\}^{4}\left\langle\gamma_{I}\right\rangle$, enables us to determine the angular-averaged second hyperpolarizability $\left\langle\gamma_{I}\right\rangle$ of the single ICT molecule. ${ }^{10}$ Here, $N$ is the number density which is obtained from the measured dilute concentration.

Table I summarizes the susceptibility $\left|\chi_{\mathrm{PR}}^{(3)}\right|=5.7$ $\times 10^{-14}$ esu obtained for the $7.04 \mathrm{mM}$ PRODAN after the subtraction of the ethanolic contribution, the susceptibility $\left|\chi_{\mathrm{ANS}}^{(3)}\right|=8.1 \times 10^{-14}$ esu for the $1.14 \mathrm{mM} 2,6$-ANS, and their corresponding second hyperpolarizabilities $\left\langle\gamma_{\mathrm{PR}}\right\rangle=4.9$ $\times 10^{-33}$ esu and $\left\langle\gamma_{\text {ANS }}\right\rangle=4.3 \times 10^{-32}$ esu, as well as their dipole-moment differences $\Delta \mu_{\mathrm{PR}}=20 \mathrm{D}$ and $\Delta \mu_{\mathrm{ANS}}=40 \mathrm{D}$. In addition, the result of the $\left\langle\gamma_{\mathrm{DE}}\right\rangle=5 \times 10^{-34} \mathrm{esu}$ and the $\Delta \mu_{\mathrm{DE}}=7.7 \mathrm{D}$ obtained by other measurements in the nonresonant region for another ICT molecule, 4- $(\mathrm{N}, \mathrm{N}-$ diethylamino)- $\beta$-nitrostyrene (DEANST), is given. ${ }^{13,14}$ The former value is the one obtained for DEANST dissolved in $\mathrm{N}$-dimethylformamide in the nonresonant region using a 


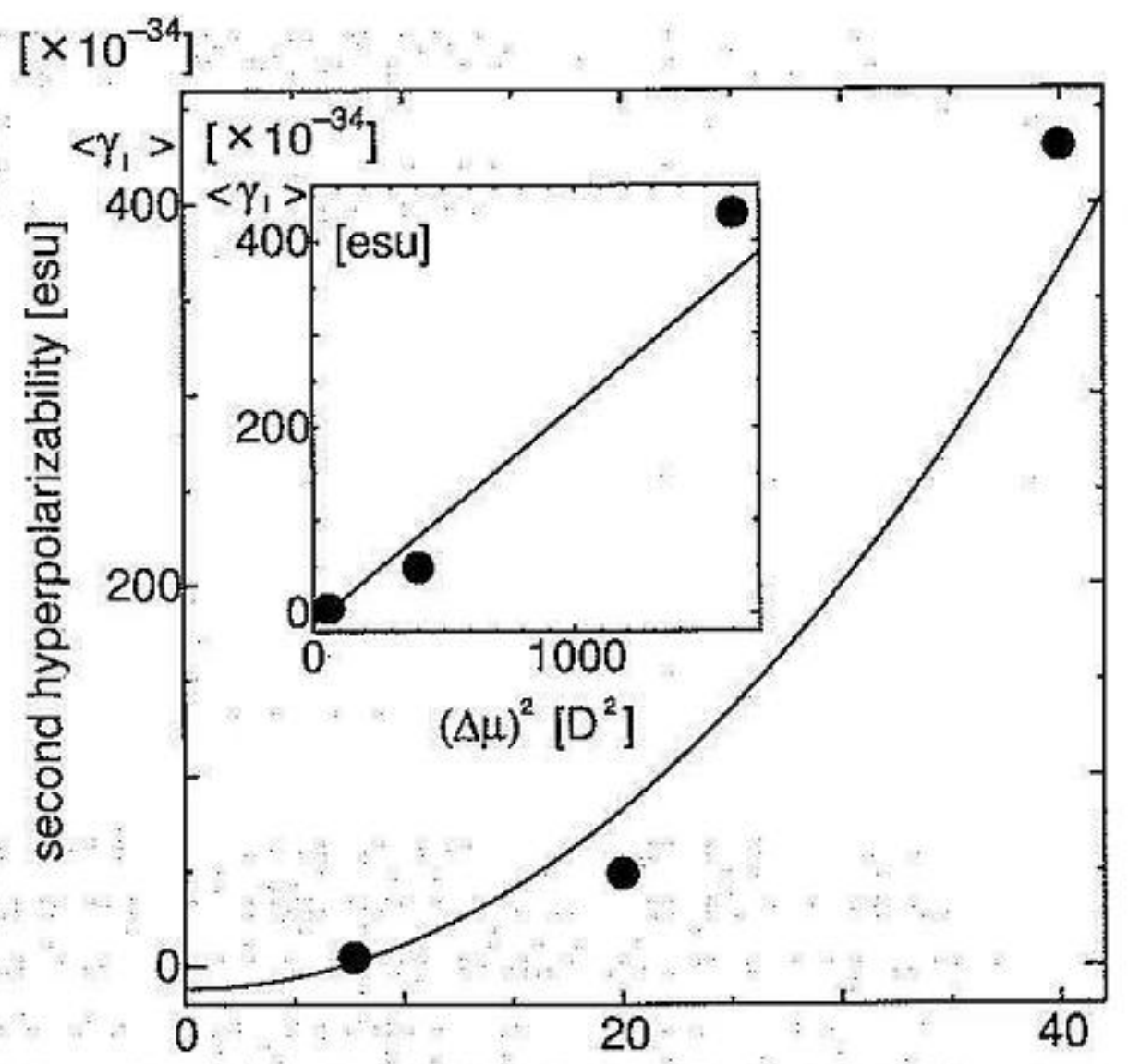

dipole moment difference $\Delta \mu$ [D]

FIG. 2. Dependence of second hyperpolarizability $\left(\left\langle\gamma_{l}\right\rangle\right)$ on permanent electric dipole-moment difference between excited and ground states $(\Delta \mu)$ for different ICT molecules. Inset, $(\Delta \mu)^{2}$ plot of $\left\langle\gamma_{l}\right\rangle$.

femtosecond time-resolved degenerate four-wave mixing spectroscopy. ${ }^{13}$ The latter value is the one obtained for DEANST doped in poly(methyl methacrylate) using an electroabsorption spectroscopy, ${ }^{14}$ which is slightly smaller than the already known one of a similar ICT molecule, 4- $(\mathrm{N}, \mathrm{N}-$ dimethylamino) $-\beta$-nitrostyrene. ${ }^{15}$ Figure 2 shows the $\Delta \mu$ dependence of the $\left\langle\gamma_{I}\right\rangle$ for different ICT molecules. It is definitely found from Fig. 2 that second hyperpolarizabilities of ICT molecules increase parabolically (solid line) as their dipole-moment differences between the excited state and the ground state increase.

There is a theoretical equation describing the relationship between the second hyperpolarizability and the permanent dipole-moment difference for the third-order nonlinearity originating from the electronic polarization in the nonresonant region. ${ }^{2,16}$ That is, the following equation obtained quantum mechanically for a quasi-two-level model using the density matrix formalism in perturbation theory is:

$$
\langle\gamma\rangle \propto \frac{1}{\left(\Delta E_{e g}\right)^{3}}\left|\mu_{g e}\right|^{2}\left[(\Delta \mu)^{2}-\left|\mu_{g e}\right|^{2}\right],
$$

where $\left|\mu_{g e}\right|$ and $\Delta E_{e g}$ represent an electronic transition dipole moment and transition energy between the excited state and the ground state, respectively. Setting Eq. (2) as $\langle\gamma\rangle$ $=a(\Delta \mu)^{2}-b$, where $b / a=\left|\mu_{g e}\right|^{2}$, permits us to fit the experimental values in Fig. 2 to a solid line (in Fig. 2) by a least-squares method, and to evaluate $\left|\mu_{g e}\right|=7.0 \mathrm{D}$. This value is reasonably compared with the transition dipole moment measured from the absorption spectrum for the typical
ICT-molecule solution $(3-7 \text { D })^{2,17}$ For example, the PRODAN/EtOH solution indicates $\left|\mu_{g e}\right|=4.5 \mathrm{D} .^{3}$ This deviation may be because the $\left|\mu_{g e}\right|$ and the $\Delta E_{e g}$ have been assumed to be not dependent on each ICT molecule. It should be noted that the quantitative result obtained by the macroscopic femtosecond experiment directly gives a clean evidence over the wide range of the dipole-moment difference for the microscopic prediction from the quantummechanical Eq. (2) for noncentrosymmetric molecules. In addition, this experimental finding provides good guidelines on the practical selection of optical materials possessing an ultrafast response $\left(\tau_{R}\right)$, a highly third-order nonlinearity $\left(\chi^{(3)}\right)$ and no absorption $(\alpha)$, that is, a large figure of merit of $\chi^{(3)} / \alpha \tau_{R}$.

In conclusion, the second hyperpolarizability due to the nonresonant electronic polarization of the ICT molecule possessing the great permanent dipole-moment difference between the excited state and the ground state has been evaluated through the measurement of the ultrafast change in refractive index for the two ICT-molecule (PRODAN and 2,6-ANS) solutions using the femtosecond time-resolved interferometer. It has been found that the second hyperpolarizability measured in the femtosecond time region rapidly increases from $5 \times 10^{-34}$ to $4.3 \times 10^{-32}$ esu with a parabolic character as the dipole-moment difference increases over the wide range from 7.7 to $40 \mathrm{D}$. Furthermore, it has been confirmed that the quantitative relationship of this experimental finding is well explained by the quantum-mechanically derived equation which describes the electronic second hyperpolarizability of one molecule possessing the permanent dipole-moment difference.

${ }^{1}$ J. L. Bredas, C. Adant, P. Tackx, A. Persoons, and B. M. Pierce, Chem. Rev. 94, 243 (1994).

${ }^{2}$ L.-T. Cheng, W. Tam, S. H. Stevenson, G. R. Meredith, G. Rikken, and S. R. Marder, J. Phys. Chem. 95, 10631 (1991).

${ }^{3}$ G. Weber and F. J. Farris, Biochemistry 18, 3075 (1979).

${ }^{4}$ C. J. Seliskar and L. Brand, J. Am. Chem. Soc. 93, 5414 (197I).

${ }^{5}$ T. Suemura, M. Ohtani, R. Morita, and M. Yamashita, Jpn. J. Appl. Phys., Part 2 36, L1307 (1997).

${ }^{6}$ K. L. Hall, A. M. Darwish, E. P. Ippen, U, Koren, and G. Raybon, Appl. Phys. Lett. 62, 1320 (1993).

${ }^{7}$ Y. Sato, R. Morita, and M. Yamashita, Jpn. J. Appl. Phys., Part 1 36, 2109 (1997).

${ }^{8}$ A. Mito, K. Hagimoto, and C. Takahashi, Nonlinear Opt. 13, 3 (1995).

${ }^{9}$ K. Kamada, M. Ueda, T. Sakaguchi, K. Ohta, and T. Fukumi, J. Opt. Soc. Am. B. 15, 838 (1998).

${ }^{10}$ R. W. Boyd, Nonlinear Optics (Academic, San Diego, 1992).

${ }^{11}$ G. M. Carter, M. K. Thakur, Y. J. Chen, and J. V. Hryniewicz, Appl. Phys. Lett. 47, 457 (1985).

${ }^{12}$ Y. Pang and P. N. Prasad, J. Chem. Phys. 93, 2201 (1990).

${ }^{13}$ Y. Yamaoka, R. Morita, and M. Yamashita, Nonlinear Opt. 22, 381 (1999).

${ }^{14} \mathrm{H}$. Murakami, R. Morita, and M. Yamashita, Nonlinear Opt. 22, 385 (1999).

${ }^{15}$ W. Liptay, Excited States, edited by E. C. Lim (Academic, New York. 1974), p. 129.

${ }^{16}$ R. Morita and M. Yamashita, Jpn. J. Appl. Phys., Part 2 32, L905 (1993)

${ }^{17}$ C. C. Teng and A. F. Garito, Phys. Rev. B 28, 6766 (1983). 